\title{
A rare case of direct inguinal hernia presented as a huge scrotal hernia in a patient with aspergillus lung disease.
}

\author{
Dr Manuneethimaran T, DrBalaji Singh .K \\ Department of general surgery, Sri ramachandra medical university, chennai, India .
}

\begin{abstract}
Direct inguinal hernia presenting as a scrotal abdomen is rare. Amongst the hernias in men, only 40 $\%$ of cases are direct. Out of these, complete scrotal hernias develop less than $5 \%$. We report a case of direct hernia that presented as a huge scrotal swelling. The patient is a case of aspergilloma of the right lung, who presented with a hernia, which was repaired with a mesh. Association of chronic cough with aspergillous lung disease might be the reason for this rare occurrence. To conclude, direct hernia can also cause large scrotal hernia especially in patients with chronic lung disease like tuberculosis, COPD and aspergillosis. Primary treatment of lung disese follwed by hernia repair with proper posterior wall strenghtening and mesh repair is the treatment protocol to be followed.
\end{abstract}

Key words:inguinal hernia, scrotal abdomen, direct hernia, aspergilloma with hernia

\section{Introduction}

Surgical management of giant inguinal hernias is difficult and often complicated. Usually scrotal inguinal hernias are indirect, where the hernial sac will be covered along with cord structures. In this report,we are discussing about abnormal presentation of direct inguinal hernia as large scrotal hernia.

\section{Case presentation}

65 years gentleman presented with a huge left side scrotal swelling with duration of 10 years. He was initialy admitted primarily to medical department for chronic cough. Onevaluation, he was diagnosed to have aspergilloma in the right lung. CT scan of the lung was done which showed solitary aspergilloma in the right lung. He was treated with medically for lung disease and transferred to surgical department for surgical management of hernia.

On clinical examination there was a large inguino scrotal swelling extending upto mid thigh. Cough impulse was present and swellingwas partialy reducible.

After complete evaluation patient was operated. Operative finding showed a huge direct sac with bowel content. Cord structure was seen separately from hernial sac. Posterior wall of inguinal canal was strengthened. Mesh repair was done. Postoperative period uneventful.

\section{Discussion}

Most of the inguinal hernias occur due to increase intra abdominal pressure secondary to chronic lung diseases, obstructive uropathy and chronic constipation. Among the chronic lung diseases chronic obstructive lung disease, tuberculosis and interstitial lung diseases are commonly associated with inguinal hernia. Rarely aspergillus lung disease is the reason for the development of hernia .In our case it is interesting that etiology of the hernia is aspergillus lung disease.

Aspergillus fumigatus classically grows in cavitated pulmonary lesions, most often in inactive tuberculous lesions, sarcoidosis lesions, lung abscess, bronchiectasis, necrotic lung cancer and cystic fibrosis. Aspergillomas are mass-like fungus balls that are typically composed of Aspergillus fumigatus, and is a noninvasive form of pulmonary aspergillosis [1]. Aspergillomas occur in patients with normal immunity but structurally abnormal lungs, with pre-existing cavities. The CT scan Appearanceof aspergilloma is a wellformed cavity with a central soft tissue attenuating rounded mass surrounded by an air. Thissign is called as crescent sign or Monod sign. The mass is ussualy spherical or ovoid [2]. The mass will be typicaly mobile in changing position of the patient. Anti mitotic drug, ltraconazole isused in high dose for aspergilloma [3].

Giant hernias dramatically impair the patient's quality of life. The affected patient's mobility will be restricted, and they often suffer from voiding difficulties as the scrotum tightens around the penis [4]. The ipsilateral testicles are atrophic or even necrotic due to presure by hernial sac. These specific problems have considerable psychological impact. These patients have serious co-morbid conditions that have an impact on both initial surgical decision-making and post-operative morbidity [5].

Indirect inguinal hernias pass through the inguinal canal via the deep ring and then superficial ring to enters the scrotum. They are present lateral to inferior epigastric vessel. 
A direct inguinal hernia protrudes through a weakened area in the transversalis fascia near the medial inguinal fossa within an anatomic region known as the inguinal or Hesselbach's triangle, an area defined by the edge of the rectus abdominis muscle, the inguinal ligament and the inferior epigastric artery. These hernias are capable of exiting via the superficial inguinal ring and are unable to extend into the scrotum [6].

In review of litrature we would found that direct hernias rarely enter the scrotum to develop huge scrotal hernias. Inetertingly, in our case a direct hernia presented as huge scrotal hernia.

\section{Conclusion}

Direct inguinal hernias presenting as a huge scrotal hernia is rare. The etiology of the hernia being an aspergilloma is another rarity. The protocol of management would be to treat the primary lung disease followed by the mesh repair of the hernia .

\section{Reference}

[1]. Anaissie EJ, McGinnis MR, Pfaller MA. Clinical Mycology. Churchill Livingstone. (2009) ISBN:1416056807

[2]. Müller NL, Franquet T, Lee KS et-al.Imaging of pulmonary infections.Lippincott Williams \& Wilkins. (2007) ISBN:078177232X.

[3]. AHMED HOSSAIN et-al,pulmonary aspergilloma, J MEDICINE 2009; 10 : 149-151

[4]. Necdet ÖZALP, Ali Osman AKTEN, Mesut TEZ Department of 5th General Surgery, Ankara Numune Teaching and Research Hospital, Ankara, Turkey.Giant scrotal hernia in an elderly man. Eur J Surg Sci 2011;2(1):23-25

[5]. Vasiliadis K, Knaebel HP, Djakovic N, Nyarangi-Dix J, Schmidt J, Büchler M. Challenging surgical management of a giant inguinoscrotal hernia: report of a case. Surg Today 2010; 40: 684-7

[6]. James Harmon M.D. Lecture 13. Human Gross Anatomy. University of Minnesota. September 4, 2008.

\section{1. consent :}

Written informed consent was obtained from the patient for publication of this case report and accompanying images. A copy of the written consent is available for review by the Editor-in-Chief of this journal on request

\section{Operative Pictures:}

Asphergilloma in the right lung
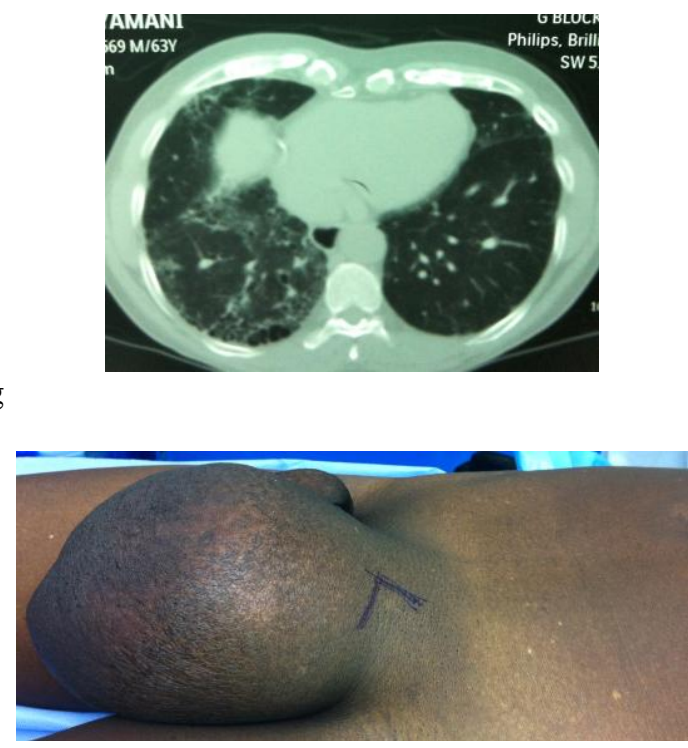

Largeleft scrotal hernia

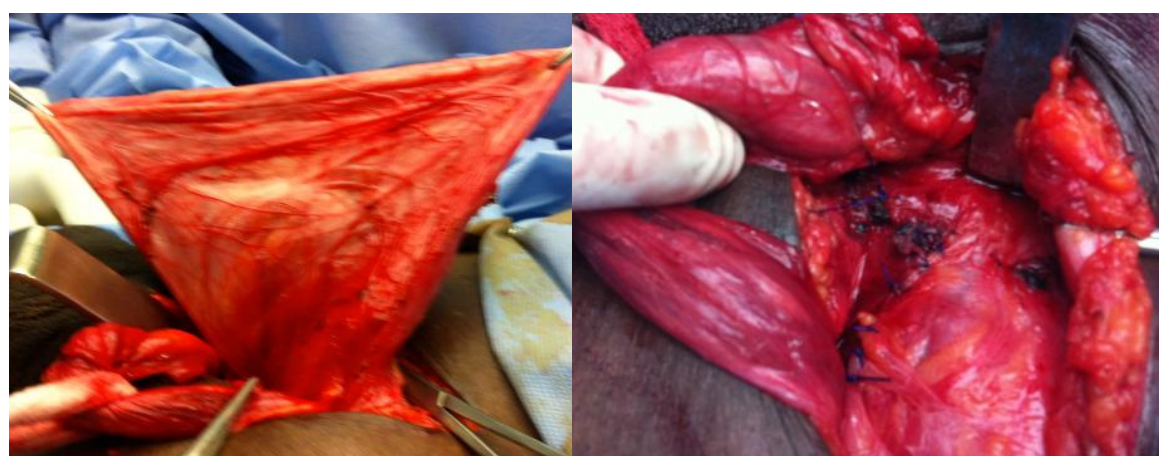

Hernial sac was separate and was medial to cord structures, which was extending medially upto pubic tubercle. After resection of excessive sac repair of the posterior wall done. 